\title{
Associations between the expression of SCCA, MTA1, P16, Ki-67 and the infection of high-risk HPV in cervical lesions
}

\author{
CUINA HAN ${ }^{1,2}$, FANGFEI ZHAO ${ }^{1}$, CHONGYANG WAN $^{1}$, YANFANG $\mathrm{HE}^{1}$ and YAN CHEN $^{1}$ \\ ${ }^{1}$ Department of Obstetrics and Gynecology, North China University of Science and Technology \\ Affiliated Hospital, Tangshan, Hebei 063000; ${ }^{2}$ Department of Obstetrics and Gynecology, \\ The Fourth Hospital of Shijiazhuang, Shijiazhuang, Hebei 050000, P.R. China
}

Received September 4, 2019; Accepted April 20, 2020

DOI: $10.3892 / \mathrm{ol} .2020 .11634$

\begin{abstract}
The application of detection technologies for human papillomavirus (HPV) has increased the resection rate for cervical intraepithelial neoplasia and early cervical cancer types. However, a large number of patients still present with advanced cervical cancer upon diagnosis. Therefore, to find a marker for the early diagnosis of cervical cancer, the present study investigated the expression profiles of squamous cell carcinoma antigen (SCCA), tumor metastasis related factor-1 (MTA1), the multiple tumor suppressor gene P16, and the nucleus-associated antigen $\mathrm{Ki}-67$ in cervical lesions, and evaluated the association between the four proteins and the infection of high-risk (HR)-HPV in cervical lesions. The rate of SCCA expression gradually increased with the progression of cervical lesions, but the increase in SCCA expression levels from low-grade squamous intraepithelial lesions (LSIL) to high-grade squamous intraepithelial lesions was not significant $(\mathrm{P}=0.197)$. The positive rate of MTA1 expression gradually increased with the development of cervical lesions, but the increase from chronic cervicitis to LSIL was not significant $(\mathrm{P}=0.258)$. The positive rates of $\mathrm{P} 16$ and Ki-67 expression exhibited significant increasing trends with the progression of cervical lesions. The expression ratio of SCCA between HR-HPV infection and non-infection groups was not statistically significant $(\mathrm{P}=0.38)$, but the expression ratios of MTA1, P16 and Ki-67 between HR-HPV infection and non-infection groups were statistically significant $(\mathrm{P}<0.05)$. These results demonstrated that the expression of SCCA, MTA1, P16 and Ki-67 increased gradually with the severity of cervical lesions. In addition, there was a positive association between the expression levels of MTA1, P16 and Ki-67 and the
\end{abstract}

Correspondence to: Professor Yan Chen, Department of Obstetrics and Gynecology, North China University of Science and Technology Affiliated Hospital, 73 Jianshe Road, Tangshan, Hebei 063000, P.R. China

E-mail: yanchen0806@yahoo.com

Key words: cervical lesions, squamous cell carcinoma antigen, metastasis-associated gene 1 , high-risk human papillomavirus infection of HR-HPV in cervical lesions. Therefore, SCCA, MTA1, P16 and Ki-67 may be used to enhance the diagnostic accuracy for cervical lesions.

\section{Introduction}

Cervical cancer is the fourth most common type of malignant cancer in women, with one of the highest incidences among all types of female genital tract malignant tumors (1). Cervical cancer is caused by a number of factors, including premature sexual activity, genetics, infection, long-term use of oral contraceptives, oral immunosuppressive drugs and poor health conditions $(2,3)$. However, it is generally accepted that the major contributing factor towards the development of cervical cancer is human papillomavirus (HPV) infection, especially high-risk (HR) HPV. HPV 16 and 18 are the most common forms of HPV (4). HPV detection has been implemented as one of the screening methods for the prevention of cervical cancer (5). With the application of HPV detection technology, the resection rate of cervical intraepithelial neoplasia and early cervical cancer has increased, but a large number of patients present with advanced cervical cancer at diagnosis (6). Therefore, to detect precancerous lesions as early as possible and reduce the incidence of cervical cancer, it is necessary to identify a biomarker for the early diagnosis of cervical cancer.

Squamous cell carcinoma antigen (SCCA) belongs to the serine/cysteine protease inhibitor family (7). As a tumor marker of squamous cell carcinomas, SCCA exhibits low expression levels in most normal tissues, but is highly expressed in malignant tumors or squamous intraepithelial lesions (8). SCCA has been extensively studied in various squamous cell carcinomas, including vulvar, esophageal and liver cancer (7-9), but also non-neoplastic diseases, such as psoriasis (10). A number of studies have demonstrated that SCCA is not only an important reference index for the diagnosis of cervical cancer types and cervical intraepithelial neoplasia, but also can provide information for the effective treatment for patients with cervical cancer (11-13). SCCA has been extensively studied in cervical lesions; however, there is no study on whether the overexpression of SCCA is associated with HPV infection.

Metastasis-associated gene 1 (MTA1) is a biomarker whose expression profile is associated with the invasive and migratory capabilities of tumor cells (14). MTA1 is expressed at low 
levels in normal tissues with the exception of the testis, but upregulated in the majority of tumor types, such as ovarian (15), breast (16), and cervical cancer (17). In the process of tumorigenesis and metastasis, MTA1 can promote the expression of tumor invasion and metastasis related factors, and inhibit the expression of tumor suppressor genes (18). MTA1 is expressed at high levels in the cervical cancer cell line $\mathrm{SiHa}$, which contains the HPV16 genome (19). The HPV E6 protein can also bind to P53 (19-21). Therefore, it is hypothesized that MTA1 upregulation in cervical lesions may be associated with HR-HPV infections. However, there is no study investigating the relationship between HR-HPV infection and MTA1 in cervical tissue, to the best of our knowledge.

The multiple tumor suppressor gene P16 directly participates in the regulation of the cell cycle (22). Upregulation of P16 in cervical lesions is associated with the expression of HPV16 and 18 genes, suggesting that the upregulation of P16 is secondary to the HPV infection (23). Therefore, P16 may be used as a marker for the differential diagnosis of benign and neoplastic hyperplasia of cervical epithelium. The nuclear associated antigen $\mathrm{Ki}-67$ is expressed in the nucleus of proliferating cells and can be used to judge the proliferative activity of cells (24). $\mathrm{Ki}-67$ is localized at the basal and parabasal layers of cervical intraepithelial neoplasia (24). The expression of Ki-67 is strongly positive in cells with a typical nucleus, and the expression profile of $\mathrm{Ki}-67$ widens and becomes more intense with the increase in the degree of cervical intraepithelial neoplasia $(25,26)$. Additionally, HR-HPV can promote $\mathrm{Ki}-67$ expression in the cervical epithelium (27). Therefore, $\mathrm{Ki}-67$ can be used as a sensitive biomarker to reflect the degree and grade of lesions. Recently, P16/Ki-67 dual staining has been used for cervical cancer screening $(28,29)$.

In the present study, the expression levels of SCCA, MTA1, P16 and Ki-67 proteins and the HR-HPV were detected in various cervical tissues. The relationship between the four proteins and the HR-HPV infection was further evaluated. The aims of the present study were to evaluate whether the trend in the expression of SCCA and MTA1 in various types of cervical lesions increased with the lesion grade and to investigate whether the expression profiles of SCCA and MTA1 were related to HR-HPV infections.

\section{Materials and methods}

Patients. The present study was approved by the Ethics Committee of the North China University of Science and Technology Affiliated Hospital, and carried out in accordance with the ethical guidelines of the World Medical Association (Declaration of Helsinki) for experiments involving humans. All patients signed informed consent for the use of their cervical tissue, secretions and clinical information.

From March 2015 to November 2016, in the North China University of Science and Technology Affiliated Hospital, 123 patients aged from 21 to 65 were enrolled in the present study, none of whom had received prior radiotherapy, chemotherapy or hormonotherapy. Of these patients, there were 31 cases with low-grade squamous intraepithelial lesions (LSILs), 31 cases with high-grade squamous intraepithelial lesions (HSILs) and 29 cases with cervical squamous cell carcinoma (CSCC). A total of 32 cases with chronic cervicitis
(CCE) were recruited as the control group. Cervical tissue was obtained from surgeries or colposcopic biopsies and immediately frozen at $80^{\circ} \mathrm{C}$ for further study. Secretions were collected using a cervical sampling brush and stored in cell preservation solution (BestBio Co., Ltd.).

Immunohistochemistry analysis. The 4\% paraformaldehyde-fixed $\left(24 \mathrm{~h}\right.$ at $\left.4^{\circ} \mathrm{C}\right)$ and paraffin-embedded tissue sections (4 $\mu \mathrm{m})$ were deparaffinized and dehydrated. For antigen retrieval, the sections were boiled in EDTA antigen retrieval solution (1:50; OriGene Technologies, Inc.) for $2.5 \mathrm{~min}$. Endogenous peroxidases and non-specific reactions were blocked by incubating the sections with $3 \% \mathrm{H}_{2} \mathrm{O}_{2}$ for $15 \mathrm{~min}$ at $37^{\circ} \mathrm{C}$ and with $10 \%$ normal goat serum (cat. no. ZLI-9022; ZSGB-BIO Co., Ltd.) for $20 \mathrm{~min}$ at $37^{\circ} \mathrm{C}$, respectively. All sections were incubated separately with the following primary antibodies: Rabbit anti-human SCCA (1:600; cat. no. A6960ABclonal Biotech Co., Ltd.), rabbit anti-human MTA1 (1:600; cat. no. A16085; ABclonal Biotech Co., Ltd.), rabbit anti-human Ki-67 (1:600; cat. no. bs-23103R; Bioss Inc.) or rabbit anti-human P16 (1:600; cat. no. bs-1856R; Bioss Inc.) at $4^{\circ} \mathrm{C}$ overnight, followed by incubation with goat anti-rabbit horseradish peroxidase labelled immunoglobulin (IgG-HRP) secondary antibodies (1:2,000, cat. no. sc2030; Santa Cruz Biotechnology Inc.) at $37^{\circ} \mathrm{C}$ for $40 \mathrm{~min}$. The staining of tissue sections was performed using a DAB Staining kit (Origene Technologies, Inc.) according to the manufacturer's instructions. Images (magnification, x200) were captured using a Micro Publisher 5.0 Confocal Microscope (Roper Technologies, Inc.) equipped with a CMOS camera (Olympus Corporation). The SCCA-, MTA1-, Ki-67- and P16-positive tissues were analyzed using CellSens Dimension software (version 1.6, Olympus Corporation).

The criteria for interpretation of the SCCA, MTA1 and P16 staining as positive were as follows: i) The presence of brown and yellow granules; ii) SCCA was located in the cytoplasm; MTA1 was in the cytoplasm, membrane and nucleus; P16 was in the cytoplasm and nucleus; iii) in each sample, the intensity of immunoreactivity (ICH) was scored as 0 (no color), 1 (light yellow), 2 (yellow) and 3 (brown), and at least 500 cells from five randomly selected staining regions were counted. The incidence of ICH was scored as 0 ( $<5 \%$ of cells stained), 1 (between 5-25\%), 2 (between 25-50\%), 3 (between 50-75\%) and $4(\geq 75 \%)$, respectively. Finally, the product of intensity and incidence was used as the criteria for the expression of protein: 0 (negative, -), 1-3 (weak positive, + ), 4-7 (moderate positive, ++ ) and 8-12 (strong positive, +++). For Ki-67, positive expression was considered as the presence of brown-yellow granules in the nucleus. The percentage of positive cells for Ki-67 was graded as previously described (30): Negative (-), <5\%; weak positive (+), 5-25\%; moderate positive $(++), 26-50 \%$; and strong positive $(+++),>50 \%$.

Western blot analysis. Total protein was obtained from cervical tissue using RIPA lysis buffer (BestBio Co., Ltd.) and quantified using a bicinchoninic acid Protein Assay kit (MultiSciences Biotech Co., Ltd.). Equal amounts of proteins ( $4 \mu 1$ per lane) were separated using 10\% SDS-PAGE (Beyotime Institute of Biotechnology) and transferred to PVDF membrane using a TRANS-BLOT SD Semidry Transfer Cell 
(Bio-Rad Laboratories, Inc.). The PVDF membrane was incubated in $5 \%$ skim milk for $1 \mathrm{~h}$ at $37^{\circ} \mathrm{C}$ and then probed with primary rabbit anti-human SCCA (1:1,000; cat. no. A6960; ABclonal Biotech Co., Ltd.) or rabbit anti-human MTA1 antibodies (1:1,000; cat. no. A16085; ABclonal Biotech Co., Ltd.) antibodies at $4^{\circ} \mathrm{C}$ overnight. After washing in $1 \mathrm{X}$ TBST buffer (Tween-20 $0.05 \%, \mathrm{v} / \mathrm{v}$ ) for $5 \mathrm{~min}$ on a shaker three times, the membrane was incubated with IgG-HRP secondary antibodies (1:2,000; cat. no. sc2030; Santa Cruz Biotechnology Inc.) for $1 \mathrm{~h}$ at $37^{\circ} \mathrm{C}$ and washed in 1X TBST buffer (Tween-20, 0.05\% v/v; cat. no. T1085; Beijing Solarbio Science \& Technology Co., Ltd.) three times. Finally, the results were visualized using the ECL Chemiluminescence Detection kit (BestBio Co., Ltd.) and signals were observed using Image Lab 5.0 (Bio-Rad Laboratories, Inc.). For quantification, SCCA and MTA1 signals were normalized against GAPDH $(1: 1,000$; cat. no. TA346930; ZSGB-BIO Co., Ltd.) and $\beta$-actin (1:1,000; cat. no. TA09; ZSGB-BIO Co., Ltd.) signals to obtain the relative expression levels of SCCA and MTA1, respectively.

HPV detection. HPV detection of cervical secretion was carried out by Cobas 4800 HPV (Roche Diagnostics), which consisted of Cobas 4800 DNA extractor (Roche Diagnostics) and Cobas z 4800 PCR-cycler (Roche Diagnostics). Relevant reagents, including sample preparation kit, HPV detection kit (PCR fluorescence method), HPV quality control kit, liquid-based cell preparation kit and PCR 96 pore plate, were purchased from Roche Diagnostics. Using a single signal, the Cobas $4800 \mathrm{HPV}$ test can not only detect HR-HPV-16 and HPV-18 specifically, but also for 12 HR-HPV types, namely 31, 33, 35, 39, 45, 51, 52, 56, 59, 66 and 68 (31). The $\beta$-globulin gene was selected as an internal control, and a Cobas 4800 real-time PCR system was applied for the PCR. Data processing was as previously described (32). The experimental conditions followed the guidelines of the manufacturer's instructions.

Statistical analysis. Statistical analyses were performed using SPSS 22.0 statistical software (IBM Corp.). The one-way ANOVA with Tukey's test was used for continuous data. Kruskal-Wallis with Dunn's post hoc test were used for comparing the results of immunohistochemistry in two different cervical lesion tissues. The relative expression quantities of SCCA and MTA1 are presented as the mean \pm SD. Dichotomous variables are presented as ratios, and comparisons between groups were performed using the $\chi^{2}$ and Fisher's exact tests. $\mathrm{P} \leq 0.05$ was considered to indicate a statistically significant difference.

\section{Results}

Expression of SCCA during the development of cervical cancer. Immunohistochemistry was used to test the expression levels of SCCA in various cervical lesion tissues. As presented in Fig. 1, positive expression of SCCA was characterized by brown and yellow granules in cytoplasm. The count and rate of positive expression of SCCA in each sample group is listed in Table I. The expression levels and staining range of SCCA increased gradually with the degree of severity of the cervical lesion and the positive rates were $12.50,45.16,54.84$ and
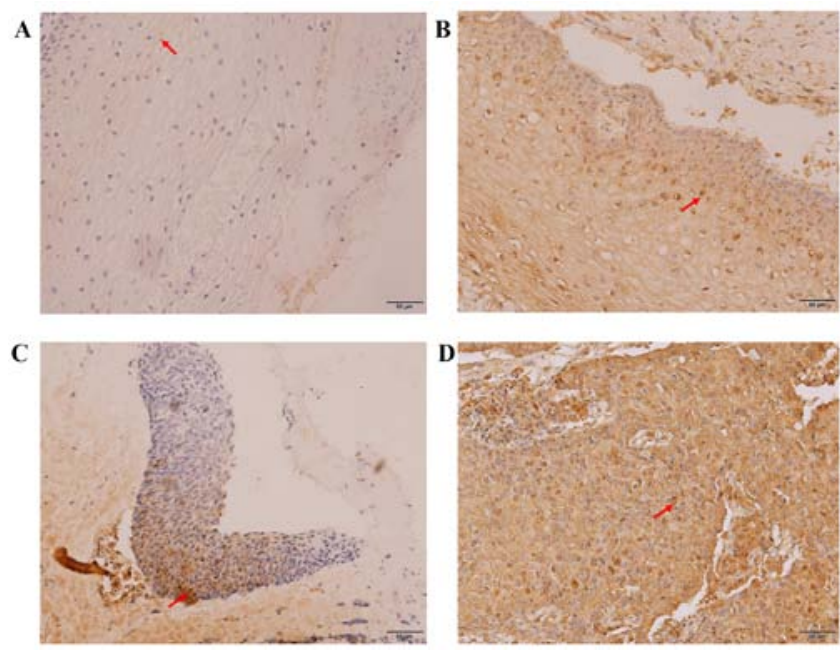

Figure 1. Immunohistochemical examination of SCCA in (A) chronic cervicitis, (B) low-grade squamous intraepithelial lesion, (C) high-grade squamous intraepithelial lesion and (D) cervical squamous cell carcinoma samples. Red arrows indicate SCCA positive staining represented by brown-yellow granules in the cytoplasm. Magnification, x200. Scale bar, $50 \mu \mathrm{m}$. SCCA, squamous cell carcinoma antigen.

89.66\% in the CCE, LSIL, HSIL and CSCC groups, respectively. Fisher's exact test demonstrated that the expression profile of SCCA was closely related to the development of cervical carcinogenesis $(\mathrm{P}<0.05)$. In addition, the number of SCCA-positive cells was lower in the CCE samples compared to the LSIL samples $(\mathrm{P}<0.008)$, as well as being lower between the HSIL samples compared to CSCC samples $(\mathrm{P}<0.008)$. However, the difference in the number of positive SCCA cells between LSIL and HSIL samples was not significant $(\mathrm{P}=0.197)$.

Western blotting was also used to examine the relative protein expression levels of SCCA in various cervical lesion tissues (Fig. 2A). The relative expression level of SCCA was strongest in the CSCC samples $(1.26 \pm 0.07)$, followed by the HSIL $(0.59 \pm 0.08)$, LSIL $(0.49 \pm 0.04)$ and CCE samples $(0.19 \pm 0.05)$ (Fig. 2B). With the degree of cervical lesions, the expression levels of SCCA gradually increased, demonstrating a significant upward trend $(\mathrm{P}<0.05)$. However, there was no significant difference in the relative expression levels of SCCA between the LSIL and HSIL samples $(P=0.13)$. This was consistent with the immunohistochemistry results.

Expression of MTA1 in the development of cervical carcinogenesis. The expression levels of MTA1 in the various cervical lesion tissues were characterized using immunohistochemistry (Fig. 3) and western blotting (Fig. 4). As presented in Fig. 3, positive expression of MTA1 was observed as brown and yellow granules in the cytoplasm, membrane and nucleus, and the expression level and staining range of MTA1 increased gradually between the CCE, LSIL, HSIL and CSCC groups. The positive rates were $9.38,19.35,58.06$ and $82.76 \%$ in the CCE, LSIL, HSIL and CSCC groups, respectively (Table II). Fisher's exact test revealed that the expression of MTA1 was associated to the development stage of cervical cancer $(\mathrm{P}<0.05)$ and that MTA1 expression presented a similar expression profile with that of SCCA. The only difference in 
Table I. Immunohistochemical examination for SCCA protein in cervical tissues from the CCE, LSIL, HSIL and CSCC groups.

\begin{tabular}{|c|c|c|c|c|c|c|c|}
\hline \multirow[b]{2}{*}{ Groups } & \multirow[b]{2}{*}{$\mathrm{n}$} & \multicolumn{4}{|c|}{ No. of specimens with IHC score } & \multirow[b]{2}{*}{ Positive rate, $\%$} & \multirow[b]{2}{*}{ P-value } \\
\hline & & - & + & ++ & +++ & & \\
\hline CCE & 32 & 28 & 4 & 0 & 0 & 12.50 & \\
\hline LSIL & 31 & 17 & 9 & 4 & 1 & 45.16 & $\begin{array}{r}<0.008^{\mathrm{a}, \mathrm{b}} \\
0.197^{\mathrm{b}, \mathrm{c}}\end{array}$ \\
\hline HSIL & 31 & 14 & 5 & 11 & 1 & 54.84 & $\begin{array}{l}<0.008^{\mathrm{a}, \mathrm{c}} \\
<0.008^{\mathrm{c}, \mathrm{d}}\end{array}$ \\
\hline $\mathrm{CSCC}$ & 29 & 3 & 2 & 8 & 16 & 89.66 & $\begin{array}{l}<0.008^{\mathrm{a}, \mathrm{d}} \\
<0.008^{\mathrm{b}, \mathrm{d}}\end{array}$ \\
\hline
\end{tabular}

${ }^{\mathrm{a}}$ vs. CCE, ${ }^{\mathrm{b}} \mathrm{vs}$. LSIL, ${ }^{\mathrm{c}}$ vs. HSIL, ${ }^{\mathrm{d}} \mathrm{vs}$. SCC; Kruskal-Wallis with Dunn's multiple comparisons test. CCE, chronic cervicitis; CSCC, cervical squamous cell carcinoma; HSIL, high-grade squamous intraepithelial lesion; IHC, immunohistochemistry; LSIL, low-grade squamous intraepithelial lesion.

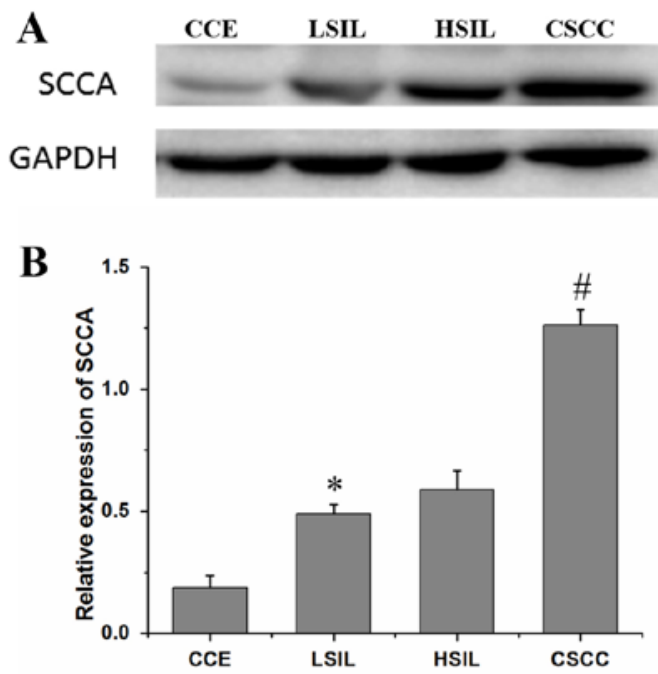

Figure 2. (A) Western blot analysis of SCCA expression levels in CCE, LSIL, HSIL and CSCC lysates. (B) The relative expression levels of SCCA (ratio of optical densities of SCCA and GAPDH) derived from western blot assays on the CCE, LSIL, HSIL and the CSCC groups. " $\mathrm{P}<0.05$ vs. CCE, ${ }^{*} \mathrm{P}<0.05$ vs. HSIL. CCE, chronic cervicitis; CSCC, cervical squamous cell carcinoma; HSIL, high-grade squamous intraepithelial lesion; LSIL, low-grade squamous intraepithelial lesion; SCCA, squamous cell carcinoma antigen.

the expression profile was that there was no significant difference in the number of positive MTA1 cells between the CCE and LSIL groups $(\mathrm{P}=0.258)$.

According to the western blotting data, the relative expression level of MTA1 was the lowest in the CCE group $(0.023 \pm 0.007)$, followed by the LSIL $(0.030 \pm 0.011)$, HSIL $(0.508 \pm 0.044)$ and CSCC $(0.707 \pm 0.076)$ groups (Fig. 4B). The expression levels of MTA1 in the late stage of cervical carcinogenesis (HSIL and CSCC) were higher compared with those in the early stage (CCE and LSIL). No significant difference was observed for the relative expression levels of MTA1 between the CCE and LSIL groups $(\mathrm{P}=0.42)$. Experimental results of the western blotting and immunohistochemical results for MTA1 were consistent.
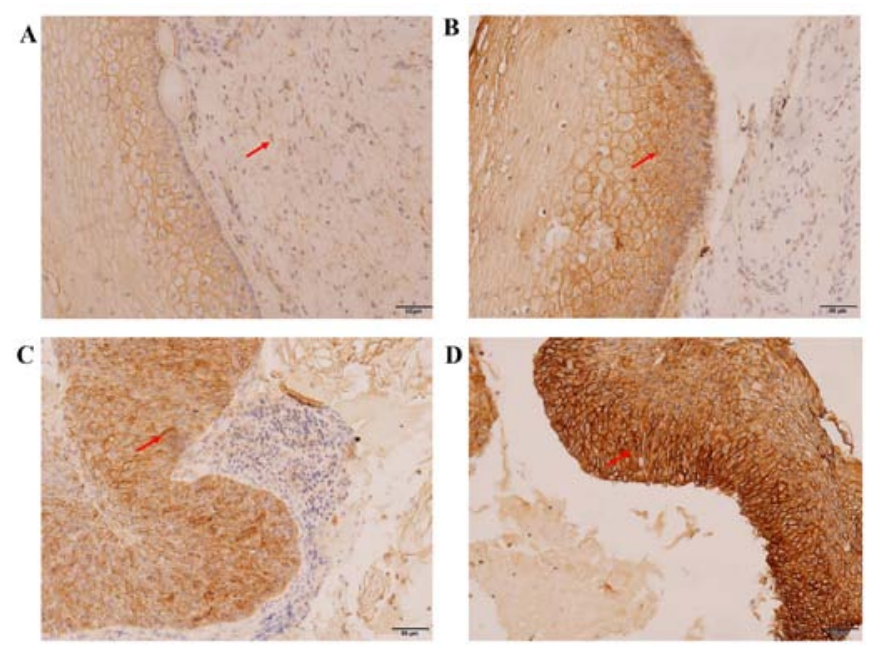

Figure 3. Immunohistochemical examination for MTA1 in (A) chronic cervicitis, (B) low-grade squamous intraepithelial lesion, (C) high-grade squamous intraepithelial lesion and (D) cervical squamous cell carcinoma samples. Red arrows indicate MTA1 positive staining in brown-yellow granules in the cytoplasm, membrane and nucleus. Magnification, x200. Scale bars represent $50 \mu \mathrm{m}$.

Expression of P16 and $\mathrm{Ki}-67$ in the development of cervical cancer. Using immunohistochemistry, positive expression of Ki-67 (Fig. 5) and P16 (Fig. 6) were characterized by the distribution of brown-yellow granules in the nucleus or the cytoplasm/nucleus, respectively. Fisher's exact test revealed that the staining range and expression levels of P16 and Ki-67 were closely associated with the degree of cervical lesion, which increased significantly with the development of cervical lesions ( $\mathrm{P} 16, \mathrm{P}<0.05$; Ki-67, $\mathrm{P}<0.05)$. The positive rates were $15.63,45.16,87.10$ and $100.00 \%$ for P16 (Table III) and 21.86, 83.87, 93.55 and $100.00 \%$ for $\mathrm{Ki}-67$ (Table IV) in the CCE, LSIL, HSIL and CSCC groups, respectively.

Expression of the four analyzed proteins and HR-HPV infection. As presented in Table V, positive rates of HR-HPV in the CCE, LSIL, HSIL and CSCC groups were 53.13, 70.97, 
A

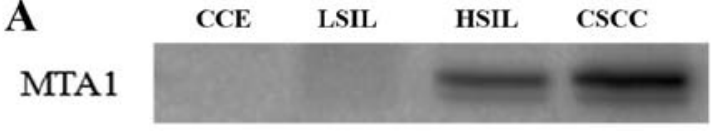

$\beta$-actin

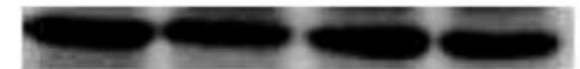

B

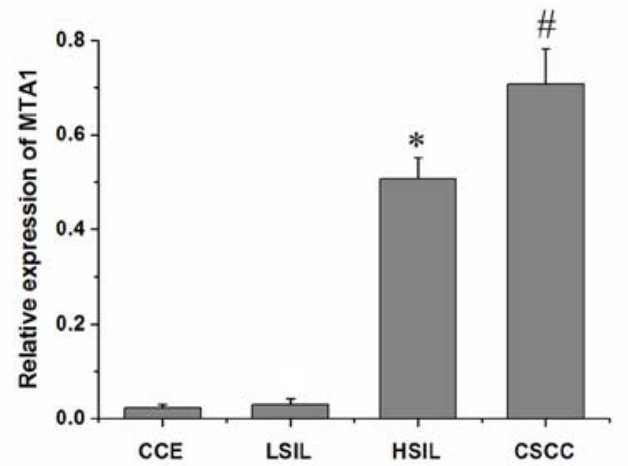

Figure 4. (A) Western blot analysis of MTA1 expression in CCE, LSIL, HSIL and CSCC. (B) The relative expression of MTA1 (ratio of optical density of SCCA to $\beta$-actin) derived from Western blot analysis of the CCE, LSIL, HSIL and CSCC groups. ANOVA, $\mathrm{P} \leq 0.05$; Tukey's tests, ${ }^{*} \mathrm{P}<0.05$ vs. LSIL, ${ }^{\#} \mathrm{P}<0.05$ vs. HSIL. CCE, chronic cervicitis; CSCC, cervical squamous cell carcinoma; HSIL, high-grade squamous intraepithelial lesion; LSIL, low-grade squamous intraepithelial lesion; MTA1, metastasis-associated gene 1; SCCA, squamous cell carcinoma antigen.

93.55 and $100.00 \%$, respectively. With the increases in the cervical lesion grade, the infection rate of HR-HPV increased significantly $(\mathrm{P}<0.05)$. However, the positive rates of HR-HPV positive samples between the CCE and LSIL groups $(\mathrm{P}=0.145)$ and between the HSIL and CSCC groups $(\mathrm{P}=0.164)$ were not statistically significant.

Fisher's exact test analysis was used to analyze the significant between the expression levels of the four proteins and the HR-HPV infection rate in cervical tissues. As demonstrated in Table VI, the expression ratio of SCCA between HR-HPV infection and non-infection groups was no statistically significant $(\mathrm{P}=0.38)$, but the expression ratios of MTA1, $\mathrm{P} 16$ and $\mathrm{Ki}-67$ between HR-HPV infection and non-infection groups were statistically significant (MTA1, $\mathrm{P}<0.05 ; \mathrm{P} 16, \mathrm{P}<0.05$; Ki-67, P<0.05).

\section{Discussion}

Cervical cancer is one of the most common gynecological tumor types that affects women (33). The pathology behind cervical cancer is caused by a variety of factors, among which HPV infection, especially HR-HPV, is currently recognized as a major pathogenic factor (4). When cervical squamous epithelial cells are infected with HPV, morphological changes occur, followed by the appearance of pre-cancerous lesions; after 5-10 years, some of these lesions develop into cervical cancer (34). The results of the present study demonstrated that the expression levels of SCCA, MTA1,P16 and Ki-67 increased with the development of cervical lesions and were associated with the HR-HPV infection status. Among the patients included in the present study, the infection rate of HR-HPV in CCE was low, but the infection rates of HR-HPV in cervical
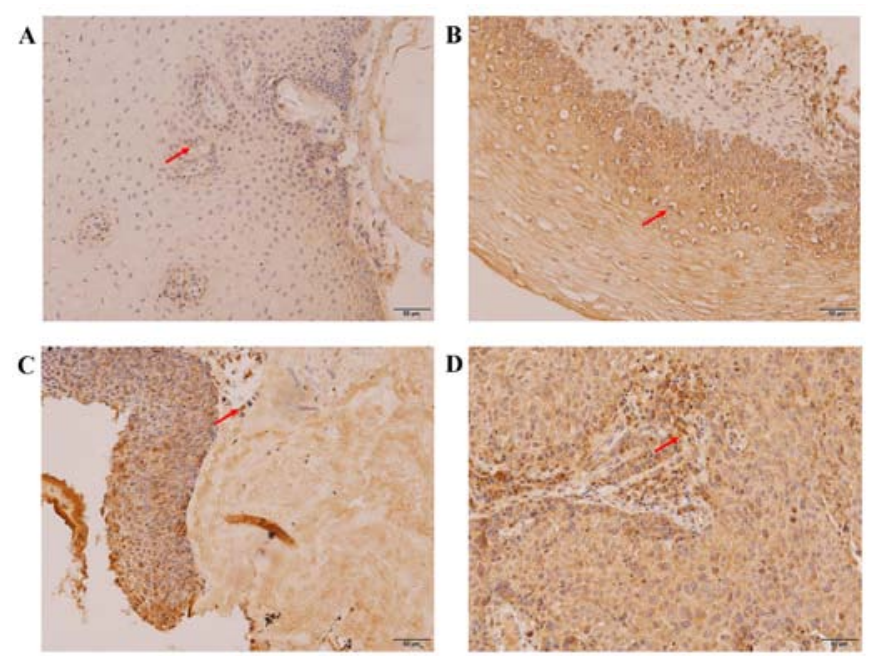

Figure 5. Immunohistochemical examination for P16 in (A) chronic cervicitis, (B) low-grade squamous intraepithelial lesion, (C) high-grade squamous intraepithelial lesion and (D) cervical squamous cell carcinoma. Red arrows indicate P16 positive staining (brown-yellow granules) in the cytoplasm and nucleus. Magnification, x200. Scale bars represent $50 \mu \mathrm{m}$.
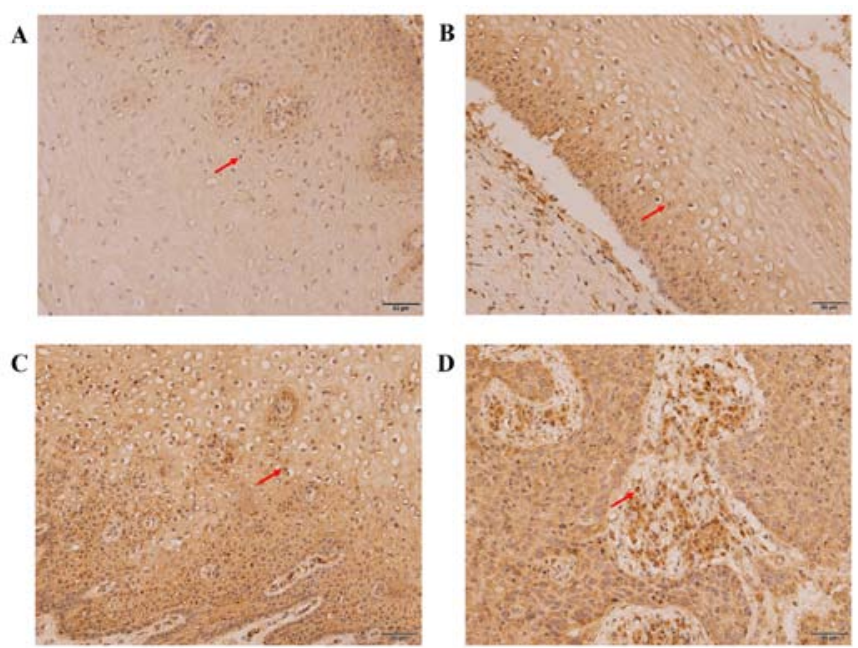

Figure 6. Immunohistochemical examination for Ki-67 (A) chronic cervicitis, (B) low-grade squamous intraepithelial lesion, (C) high-grade squamous intraepithelial lesion and (D) cervical squamous cell carcinoma. Red arrows indicate Ki-67 positive staining (brown-yellow granules) in the nucleus. Magnification, $\mathrm{x} 200$. Scale bar, $50 \mu \mathrm{m}$.

pre-cancerous lesions (LSIL and HSIL) and cervical cancer (CSCC) were high, which was similar to a previous report (35). Therefore, HPV infection is associated with the occurrence of cervical cancer.

The expression levels of P16 and Ki-67 gradually increase with the development of cervical intraepithelial lesions and cervical cancer $(23,25,26)$. Consequently, P16/Ki-67 dual staining has been used for cervical cancer screening $(28,29)$. In this present study, the positive rates of $\mathrm{P} 6$ and $\mathrm{Ki}-67$ in the CCE, LSIL, HSIL and CSCC samples exhibited a significant increasing trend: Their expression levels in the LSIL, HSIL and CSCC samples were significantly higher compared with those in CCE; the expression levels in the CSCC samples were significantly higher compared with those in the HSIL and LSIL samples; and the expression levels in the HSIL 
Table II. Immunohistochemical examination for metastasis-associated gene 1 protein in cervical tissue from the CCE, LSIL, HSIL and CSCC groups.

\begin{tabular}{|c|c|c|c|c|c|c|c|}
\hline \multirow[b]{2}{*}{ Groups } & \multirow[b]{2}{*}{$\mathrm{n}$} & \multicolumn{4}{|c|}{$\begin{array}{l}\text { Number of specimens } \\
\text { with different IHC scores }\end{array}$} & \multirow[b]{2}{*}{ Positive rate $(\%)$} & \multirow[b]{2}{*}{ P-value } \\
\hline & & - & + & ++ & +++ & & \\
\hline CCE & 32 & 29 & 2 & 1 & 0 & 9.38 & \\
\hline LSIL & 31 & 25 & 4 & 1 & 1 & 19.35 & $\begin{array}{r}0.258^{\mathrm{a}, \mathrm{b}} \\
<0.008^{\mathrm{b}, \mathrm{c}}\end{array}$ \\
\hline HSIL & 31 & 13 & 3 & 11 & 4 & 58.06 & $\begin{array}{l}<0.008^{\mathrm{a}, \mathrm{c}} \\
<0.008^{\mathrm{c}, \mathrm{d}}\end{array}$ \\
\hline $\mathrm{CSCC}$ & 29 & 5 & 2 & 6 & 16 & 82.76 & $\begin{array}{l}<0.008^{\mathrm{a}, \mathrm{d}} \\
<0.008^{\mathrm{b}, \mathrm{c}}\end{array}$ \\
\hline
\end{tabular}

${ }^{\mathrm{a}}$ vs. CCE, ${ }^{b}$ vs. LSIL, ${ }^{\mathrm{c}}$ vs. HSIL, ${ }^{\mathrm{d}} \mathrm{vs}$. SCC; Kruskal-Wallis with Dunn's multiple comparisons test. CCE, chronic cervicitis; CSCC, cervical squamous cell carcinoma; HSIL, high-grade squamous intraepithelial lesion; IHC, immunohistochemistry; LSIL, low-grade squamous intraepithelial lesion.

Table III. Immunohistochemical examination for P16 protein in cervical tissues from the CCE, LSIL, HSIL and CSCC groups.

\begin{tabular}{|c|c|c|c|c|c|c|c|}
\hline \multirow[b]{2}{*}{ Groups } & \multirow[b]{2}{*}{$\mathrm{n}$} & \multicolumn{4}{|c|}{$\begin{array}{l}\text { Number of specimens } \\
\text { with different IHC score }\end{array}$} & \multirow[b]{2}{*}{ Positive rate $(\%)$} & \multirow[b]{2}{*}{ P-value } \\
\hline & & - & + & ++ & +++ & & \\
\hline CCE & 32 & 27 & 4 & 1 & 0 & 15.63 & \\
\hline LSIL & 31 & 17 & 9 & 3 & 2 & 45.16 & $\begin{array}{r}0.009^{\mathrm{a}, \mathrm{b}} \\
<0.008^{\mathrm{b}, \mathrm{c}}\end{array}$ \\
\hline HSIL & 31 & 4 & 5 & 7 & 15 & 87.10 & $\begin{array}{l}<0.008^{\mathrm{a}, \mathrm{c}} \\
<0.008^{\mathrm{c}, \mathrm{d}}\end{array}$ \\
\hline $\mathrm{CSCC}$ & 29 & 0 & 1 & 4 & 24 & 100.0 & $\begin{array}{l}<0.008^{\mathrm{a}, \mathrm{d}} \\
<0.008^{\mathrm{b}, \mathrm{d}}\end{array}$ \\
\hline
\end{tabular}

${ }^{a}$ vs. CCE, ${ }^{b}$ vs. LSIL, ${ }^{c}$ vs. HSIL, ${ }^{d}$ vs. SCC; Kruskal-Wallis with Dunn's multiple comparisons test. CCE, chronic cervicitis; CSCC, cervical squamous cell carcinoma; HSIL, high-grade squamous intraepithelial lesion; IHC, immunohistochemistry; LSIL, low-grade squamous intraepithelial lesion.

samples were significantly higher compared with those in the LSIL samples. The results were consistent with previous reports $(23,25,26)$. With the increase of P16 and Ki67 expression levels, the number of HR-HPV copies increases (34). The results of the present study revealed that the expression of P16 and $\mathrm{Ki}-67$ were positively associated with the HR-HPV infection status. Therefore, upregulation of P16 and Ki-67 indicated infection with HR-HPV. This observation may provide a basis for the early diagnosis of cervical lesions and was consistent with a previous report $(34,36)$.

As a specific antigen produced by squamous cell carcinomas, SCCA has been studied extensively in cervical cancer (8-13). Although it has been confirmed that SCCA expression is highly associated with the occurrence and development of CVCC $(8,12,13)$, there are limited reports investigating the expression of SCCA in cervical pre-cancerous lesions. In addition, HPV, especially high-risk
HPV, is the main cause of cervical lesions (4). However, to the best of our knowledge, there are no studies investigating the relationship between SCCA and HPV infections. In this present study, the expression levels of SCCA in the LSIL, HSIL and CSCC samples were significantly higher compared with those in the CCE samples. The expression of SCCA in the CSCC samples was significantly higher compared with that in the HSIL and LSIL samples, and the expression of SCCA in the HSIL samples was higher compared with that in the LSIL samples; however, there was no significant difference between the HSIL and LSIL samples. The expression levels of SCCA increased with the development of cervical cancer, which indicated that SCCA may have a clinical role in the diagnosis of cervical intraepithelial neoplasia and cervical cancer. Combining SCCA and P16/Ki-67 dual staining may be used to improve the accuracy of disease diagnosis, especially for distinguishing HSIL and CSCC; therefore, it may help to 
Table IV. Immunohistochemical examination for Ki-67 protein in cervical tissues from the CCE, LSIL, HSIL and CSCC groups.

\begin{tabular}{|c|c|c|c|c|c|c|c|}
\hline \multirow[b]{2}{*}{ Groups } & \multirow[b]{2}{*}{$\mathrm{n}$} & \multicolumn{4}{|c|}{$\begin{array}{l}\text { Number of specimens } \\
\text { with different IHC score }\end{array}$} & \multirow[b]{2}{*}{ Positive rate $(\%)$} & \multirow[b]{2}{*}{ P-value } \\
\hline & & - & + & ++ & +++ & & \\
\hline $\mathrm{CCE}$ & 32 & 25 & 6 & 1 & 0 & 21.86 & \\
\hline LSIL & 31 & 5 & 19 & 6 & 1 & 83.87 & $\begin{array}{l}<0.008^{\mathrm{a}, \mathrm{b}} \\
<0.008^{\mathrm{b}, \mathrm{c}}\end{array}$ \\
\hline HSIL & 31 & 2 & 6 & 9 & 14 & 93.55 & $\begin{array}{l}<0.008^{\mathrm{a}, \mathrm{c}} \\
<0.008^{\mathrm{c}, \mathrm{d}}\end{array}$ \\
\hline CSCC & 29 & 0 & 0 & 2 & 27 & 100.0 & $\begin{array}{l}<0.008^{\mathrm{a}, \mathrm{d}} \\
<0.008^{\mathrm{b}, \mathrm{d}}\end{array}$ \\
\hline
\end{tabular}

${ }^{\mathrm{a}}$ vs. CCE, ${ }^{\mathrm{b} v s .}$ LSIL, ${ }^{\mathrm{c}}$ vs. HSIL, dvs. SCC; Kruskal-Wallis with Dunn's multiple comparisons test. CCE, chronic cervicitis; CSCC, cervical squamous cell carcinoma; HSIL, high-grade squamous intraepithelial lesion; IHC, immunohistochemistry; LSIL, low-grade squamous intraepithelial lesion.

Table V. Infection rates of HR-HPV in cervical tissues from the CCE, LSIL, HSIL and CSCC groups.

\begin{tabular}{|c|c|c|c|c|c|c|}
\hline \multirow[b]{2}{*}{ Groups } & \multirow[b]{2}{*}{$\mathrm{n}$} & \multicolumn{2}{|c|}{ HR-HPV (+) } & \multicolumn{2}{|c|}{ HR-HPV (-) } & \multirow[b]{2}{*}{ P-value } \\
\hline & & $\mathrm{n}_{1}$ & Positive \% & $\mathrm{n}_{2}$ & Negative $\%$ & \\
\hline $\mathrm{CCE}$ & 32 & 17 & 53.13 & 15 & 46.87 & \\
\hline LSIL & 31 & 22 & 70.97 & 9 & 29.03 & $\begin{array}{r}0.145^{\mathrm{a}, \mathrm{b}} \\
<0.008^{\mathrm{b}, \mathrm{c}}\end{array}$ \\
\hline HSIL & 31 & 29 & 93.55 & 2 & 6.45 & $\begin{array}{l}0.020^{\mathrm{a}, \mathrm{c}} \\
0.164^{\mathrm{c}, \mathrm{d}}\end{array}$ \\
\hline $\mathrm{CSCC}$ & 29 & 29 & 100.0 & 0 & 0.00 & $\begin{array}{l}<0.008^{\mathrm{a}, \mathrm{d}} \\
<0.008^{\mathrm{b}, \mathrm{d}}\end{array}$ \\
\hline
\end{tabular}

${ }^{\mathrm{a}}$ vs. CCE, ${ }^{\mathrm{b}}$ vs. LSIL, ${ }^{\mathrm{c}}$ vs. HSIL, ${ }^{\mathrm{d}} \mathrm{vs}$. SCC; $\chi^{2}$ test. CCE, chronic cervicitis; CSCC, cervical squamous cell carcinoma; HR-HPV, high-risk human papillomavirus; HSIL, high-grade squamous intraepithelial lesion; LSIL, low-grade squamous intraepithelial lesion.

Table VI. The expression of the four analyzed proteins and infection of HR-HPV in cervical tissues.

\begin{tabular}{lcrrrrr}
\hline & \multicolumn{5}{c}{ Number of specimens } \\
& & \multicolumn{5}{c}{ with IHC score } \\
\cline { 3 - 6 } Variable & HR-HPV & - & + & ++ & +++ & P-value \\
\hline \multirow{2}{*}{ SCCA } & - & 15 & 6 & 3 & 2 & 0.38 \\
& + & 47 & 14 & 20 & 16 & \\
MTA1 & - & 19 & 4 & 2 & 1 & $<0.05$ \\
\multirow{2}{*}{ P16 } & + & 53 & 7 & 17 & 20 & \\
\multirow{2}{*}{ Ki-67 } & - & 15 & 7 & 3 & 1 & $<0.05$ \\
& + & 33 & 12 & 12 & 40 & \\
& + & 14 & 7 & 3 & 2 & $<0.05$ \\
& + & 18 & 24 & 15 & 40 &
\end{tabular}

Fisher's exact test was performed. HR-HPV, high-risk human papillomavirus; IHC, immunohistochemistry; MTA1, metastasis-associated gene 1; SCCA, squamous cell carcinoma antigen. ensure that appropriate treatment is provided for patients with cervical cancer. No association was observed between SCCA expression levels and HR-HPV infection in various cervical lesions $(P=0.20)$ in the present study, which may be due to the small sample size.

MTA1 is a gene associated with tumor metastasis and has been extensively studied in the field of gynecological cancer $(14,15,17)$. Abnormal expression of MTA1 is not only related to the occurrence and metastasis of ovarian cancer, but is also associated with the clinical stage of ovarian cancer (15). The expression levels of MTA1 in cervical cancer have been demonstrated to be higher compared with those in the normal cervical tissue, and upregulation of MTA1 is positively correlated with the clinical stage and lymph node metastasis in patients with cervical cancer (17). However, the expression of MTA1 in cervical lesions has been not reported. In this present study, the expression intensity of MTA1 was associated with the degree of the cervical lesions. The expression of MTA1 in the CSCC samples was significantly higher compared with that in the LSIL, HSIL and CCE samples; the expression of 
MTA1 in the HSIL samples also appeared higher compared with that in the CCE samples, but no significant difference was observed. In addition, the expression of MTA1 in cervical tissue was associated with the HR-HPV infection status. Previous studies have demonstrated that MTA1 can interact with P53 (19-21). It is assumed that the HPV E6 protein may interact with P53 after infection with HR-HPV and that the decrease of P53 may provide a feedback stimulation leading to raised protein expression levels of MTA1 $(20,21)$. In addition, the expression of MTA1 in various cervical lesions was consistent with that of P16 and Ki-67. Upregulation of MTA1 was associated with infection with HR-HPV; therefore, MTA1 may be a useful biomarker for the early diagnosis of cervical lesions.

The main limitation to this study was that the relationship between the development of cervical lesions and the blood concentration of the four proteins was not determined. However, SCCA, MTA1, P16 and Ki-67 may indicate the occurrence and development of cervical lesions. The mechanism of action behind their specific roles needs to be further studied, which will lay a theoretical foundation for the early diagnosis and effective treatment of cervical lesions in the clinic.

In conclusion, SCCA, MTA1, P16 and Ki-67 were upregulated in cervical lesions and their positive rates increased with the development of cervical lesions. Furthermore, the expression levels of MTA1, P16 and Ki-67 were associated with the infection rate of HR-HPV. SCCA and MTA1 may be used for cervical cancer screening in a similar manner to $\mathrm{P} 16 / \mathrm{Ki}-67$ double staining. In particular, SCCA may be used to distinguish between patients with HSIL and CSCC, and MTA1 can be used to distinguish between those with LSIL and HSIL.

\section{Acknowledgements}

Not applicable.

\section{Funding}

Not applicable.

\section{Availability of data and materials}

The datasets used and/or analyzed during the current study are available from the corresponding author on reasonable request.

\section{Authors' contributions}

$\mathrm{CH}$ and $\mathrm{YC}$ conceived and designed the study. $\mathrm{CH}, \mathrm{FZ}$ and $\mathrm{CW}$ performed the experiments. $\mathrm{CH}$ and $\mathrm{YH}$ analyzed the data. $\mathrm{CH}$ and $\mathrm{YC}$ wrote the manuscript. All authors read and approved the final manuscript.

\section{Ethics approval and consent to participate}

The present study was approved by the ethnic committee of the North China University of Science and Technology Affiliated Hospital. Informed consent was obtained from all patients.

\section{Patient consent for publication}

Not applicable.

\section{Competing interests}

The authors declare that they have no competing interests.

\section{References}

1. Arbyn M, Weiderpass E, Bruni L, de Sanjosé S, Saraiya M, Ferlay $\mathbf{J}$ and Bray F: Estimates of incidence and mortality of cervical cancer in 2018: A worldwide analysis. Lancet Glob Health 8: e191-e203, 2020.

2. Plummer M, Peto J and Franceschi S; International Collaboration of Epidemiological Studies of Cervical Cancer: Time since first sexual intercourse and the risk of cervical cancer. Int J Cancer 130: 2638-2644, 2012.

3. Shrestha AD, Neupane D, Vedsted P and Kallestrup P: Cervical cancer prevalence, incidence and mortality in low and middle income countries: A systematic review. Asian Pac J Cancer Prev 19: 319-324, 2018.

4. Arbyn M, Snijders PJ, Meijer CJ, Berkhof J, Cuschieri K, Kocjan BJ and Poljak M: Which high-risk HPV assays fulfil criteria for use in primary cervical cancer screening? Clin Microbiol Infect 21: 817-826, 2015.

5. Wright TC, Stoler MH, Behrens CM, Sharma A, Zhang G and Wright TL: Primary cervical cancer screening with human papillomavirus: End of study results from the ATHENA study using HPV as the first-line screening test. Gynecol Oncol 136: 189-197, 2015.

6. Dasari S, Wudayagiri R and Valluru L: Cervical cancer: Biomarkers for diagnosis and treatment. Clin Chim Acta 445: 7-11, 2015.

7. Pozzan C, Cardin R, Piciocchi M, Cazzagon N, Maddalo G, Vanin V, Giacomin A, Pontisso P, Cillo U and Farinati F: Diagnostic and prognostic role of SCCA-IgM serum levels in hepatocellular carcinoma (HCC). J Gastroenterol Hepatol 29: 1637-1644, 2014

8. Maddalo G, Fassan M, Cardin R, Piciocchi M, Marafatto F, Rugge M, Zaninotto G, Pozzan C, Castoro C, Ruol A, et al: Squamous cellular carcinoma antigen serum determination as a biomarker of barrett esophagus and esophageal cancer. J Clin Gastroenterol 52: 401-406, 2018.

9. Chechlinska M, Kowalewska M, Brzoska-Wojtowicz E, Radziszewski J, Ptaszynski K, Rys J, Kaminska J and Nowak R: Squamous cell carcinoma antigen 1 and 2 expression in cultured normal peripheral blood mononuclear cells and in vulvar squamous cell carcinoma. Tumor Biol 31: 559-567, 2010

10. El-Rachkidy RG, Young HS, Griffiths CE and Camp RD: Humoral autoimmune responses to the squamous cell carcinoma antigen protein family in psoriasis. J Invest Dermatol 128: 2219-2224, 2008.

11. Jeong BK, Choi DH, Huh SJ, Park W, Bae DS and Kim BG: The role of squamous cell carcinoma antigen as a prognostic and predictive factor in carcinoma of uterine cervix. Radiat Oncol J 29: 191-198, 2011.

12. Shimura K. Mabuchi S, Yokoi T, Sasano T, Sawada K, Hamasaki T and Kimura T: Utility of serum squamous cell carcinoma antigen levels at the time of recurrent cervical cancer diagnosis in determining the optimal treatment choice. J Gynecol Oncol 24: 321-329, 2013.

13. Kim BG: Squamous cell carcinoma antigen in cervical cancer and beyond. J Gynecol Oncol 24: 291-292, 2013.

14. Sen N, Gui B and Kumar R: Role of MTA1 in cancer progression and metastasis. Cancer Metastasis Rev 33: 879-889, 2014.

15. Yi S, Guangqi $\mathrm{H}$ and Guoli $\mathrm{H}$ : The association of the expression of MTA1, NM23H1 with the invasion, metastasis of ovarian carcinoma. Chin Med Sci J 18: 87-92, 2003.

16. Guddeti RK, Bali P, Karyala P and Pakala SB: MTA1 coregulator regulates LDHA expression and function in breast cancer. Biochem Biophys Res Commun 520: 54-59, 2019.

17. Bilikezi-Aikemu F, Min L, Xiumei Z, Junling G and Danjin G, Qi: Expression and significance of MTA1, MMP-2, MMP-7 in cervical cancer. Chin J Mod Med 23: 51-54, 2013. 
18. Dannenmann C, Shabani N, Friese K, Jeschke U, Mylonas I and Brüning A: The metastasis-associated gene MTA1 is upregulated in advanced ovarian cancer, represses ER $\beta$, and enhances expression of oncogenic cytokine GRO. Cancer Biol Ther 7: 1460-1467, 2008.

19. Rao Y, Wang H, Fan L and Chen G: Silencing MTA1 by RNAi reverses adhesion, migration and invasiveness of cervical cancer cells ( $\mathrm{SiHa}$ ) via altered expression of $\mathrm{p} 53$, and E-cadherin/ $\beta$-catenin complex. J Huazhong Univ Sci Technolog Med Sci 31: 1-9, 2011.

20. Scheffner M, Werness BA, Huibregtse JM, Levine AJ and Howley PM: The E6 oncoprotein encoded by human papillomavirus types 16 and 18 promotes the degradation of p53. Cell 63: 1129-1136, 1990.

21. Moody CA and Laimins LA: Human papillomavirus oncoproteins: Pathways to transformation. Nat Rev Cancer 10: 550-560, 2010.

22. Pan FP, Zhou HK, Bu HQ, Chen ZQ, Zhang H, Xu LP, Tang J, Yu QY, Chu YQ, Pan J, et al: Emodin enhances the demethylation by 5 -Aza-CdR of pancreatic cancer cell tumor-suppressor genes P16, RASSF1A and ppENK. Oncol Rep 35: 1941-1949, 2016.

23. Bleotu C, Botezatu A, Goia CD, Socolov D, Corniţescu F, Teleman S, Huică I, Iancu I and Anton G: P16INK4A-A possible marker in HPV persistence screening. Roum Arch Microbiol Immunol 68: 183-189, 2009.

24. Sun $X$ and Kaufman PD: Ki-67: More than a proliferation marker. Chromosoma 127: 175-186, 2018

25. Kimura M, Matsumoto T, Morizane T, Sonoue H, Ogishima D and Kinoshita K: Histopathological study of the spreading neoplastic cells in cervical glands and surface epithelia in cervical intra-epithelial neoplasia and microinvasive squamous cell carcinoma: Ki-67 immunostaining is a useful marker for pathological diagnosis from the gland involvement site. Pathol Int 56: 428-433, 2006.

26. Carreras R, Alameda F, Mancebo G, García-Moreno P, Mariñoso ML, Costa C, Fusté P, Baró T and Serrano S: A study of $\mathrm{Ki}-67, \mathrm{c}-$ erbB2 and cyclin D-1 expression in CIN-I, CIN-III and squamous cell carcinoma of the cervix. Histol Histopathol 22: 587-592, 2007.

27. Yang D, Xu Q and Mao Q: Expression of P16, Ki67 and HPV16/18 in cervical lesions and their clinical implications. Int J Pathol Clin Med 32: 211-215, 2012.

28. Clarke MA, Cheung LC, Castle PE, Schiffman M, Tokugawa D, Poitras N, Lorey T, Kinney W and Wentzensen N: Five-year risk of cervical precancer following p16/ki-67 dual-stain triage of HPV-positive women. JAMA Oncol 5: 181-186, 2019.
29. Wentzensen N, Clarke MA, Bremer R, Poitras N, Tokugawa D, Goldhoff PE, Castle PE, Schiffman M, Kingery JD, Grewal KK, et al: Clinical evaluation of human papillomavirus screening with $\mathrm{p} 16 / \mathrm{ki}-67$ dual stain triage in a large organized cervical cancer screening program. JAMA Intern Med 179: 881-888, 2019.

30. Zhong P, Li J, Gu Y, Liu Y, Wang A, Sun Y and Lu L: P16 and Ki-67 expression improves the diagnostic accuracy of cervical lesions but not predict persistent high risk human papillomavirus infection with CIN1. Int J Clin Exp Pathol 8: 2979-2986, 2015.

31. Pesic A, Krings A, Hempel M, Preyer R, Chatzistamatiou K, Agorastos T and Kaufmann AM: CIN2+ detection of the HPV DNA array genotyping assay in comparison with the cobas 4800 HPV test and cytology. Virol J 16: 92, 2019.

32. Tang Z, Xu Y, Song N, Zou D, Liao Y, Li Q and Pan C: A comparison of the MeltPro® HPV test with the Cobas ${ }^{\circledR}$ HPV test for detecting and genotyping 14 high-risk human papillomavirus types. Arch Virol 163: 725-730, 2018.

33. Brusselaers N, Shrestha S, van de Wijgert J and Verstraelen H: Vaginal dysbiosis and the risk of human papillomavirus and cervical cancer: Systematic review and meta-analysis. Am J Obstet Gynecol 221: 9-18, 2019.

34. Melnikow J, Henderson JT, Burda BU, Senger CA, Durbin SS and Weyrich MS: Screening for cervical cancer with high-risk human papillomavirus testing. JAMA 320: 687-705, 2018.

35. Kjær SK, Munk C, Junge J and Iftner T: Carcinogenic HPV prevalence and age-specific type distribution in 40,382 women with normal cervical cytology, ASCUS/LSIL, HSIL, or cervical cancer: What is the potential for prevention? Cancer Causes Control 25: 179-189, 2014.

36. Song SH, Park HM, Eom DW, Lee JK, Lee NW, Kim AR, Hur JY, Lee KW, Park YK and Saw HS: The expression of p16 (INK4a) and $\mathrm{Ki}-67$ in relation to high-risk human papilloma viral load and residual disease after conization with positive margins. Int J Gynecol Cancer 17: 858-867, 2007.

This work is licensed under a Creative Commons Attribution-NonCommercial-NoDerivatives 4.0 International (CC BY-NC-ND 4.0) License. 